\title{
Establishment of Mouse Models of Psoriasis with Blood Stasis Syndrome Complicated with Glucose and Lipid Metabolism Disorders
}

\author{
Ying Luo, ${ }^{1}$ Yi Ru, ${ }^{1}$ Huaibo Zhao, ${ }^{1}$ Liu Liu, ${ }^{1}$ Seokgyeong Hong, ${ }^{1}$ Xiaoying Sun, ${ }^{2}$ Le Kuai, \\ Yi Lu, ${ }^{1}$ Meng Xing, ${ }^{1}$ Xi Chen, ${ }^{1}$ Jiankun Song, ${ }^{1}$ Yue Luo, ${ }^{1}$ Xiaoya Fei, ${ }^{1}$ Yaqiong Zhou, \\ Hongjin $\mathrm{Li}^{2}{ }^{2} \mathrm{Bin} \mathrm{Li}\left(\mathbb{D},^{1,2}\right.$ and $\mathrm{Xin} \mathrm{Li}{ }^{1,2}$ \\ ${ }^{1}$ Department of Dermatology, Yueyang Hospital of Integrated Traditional Chinese and Western Medicine, \\ Shanghai University of Traditional Chinese Medicine, Shanghai 200437, China \\ ${ }^{2}$ Institute of Dermatology, Shanghai Academy of Traditional Chinese Medicine, Shanghai 201203, China \\ Correspondence should be addressed to Bin Li; 18930568129@163.com and Xin Li; 13661956326@163.com
}

Received 3 August 2019; Accepted 21 October 2019; Published 12 November 2019

Academic Editor: Adolfo Andrade-Cetto

Copyright (C) 2019 Ying Luo et al. This is an open access article distributed under the Creative Commons Attribution License, which permits unrestricted use, distribution, and reproduction in any medium, provided the original work is properly cited.

\begin{abstract}
Background. Psoriasis has been reported as a high-risk factor for quality of life and survival rate in patients with metabolic disorder. However, there is no animal model for studying this disease. This study aimed to establish and evaluate mouse models of psoriasis with blood stasis syndrome (which is a key to psoriasis pathogenesis, according to Chinese Medicine) complicated with metabolic disorders. Method. Forty-five C57BL/6 mice were randomly divided into the blank control (Control), psoriasis (Imiquimod (IMQ)), psoriasis with metabolic disorders (IMQ + streptozotocin (STZ)), psoriasis with blood stasis syndrome (BSS) (IMQ + BSS), and psoriasis with blood stasis syndrome complicated with metabolic disorders (IMQ + STZ + BSS) groups $(n=9$ mice/group). Psoriasis lesions were induced using IMQ cream (on both the ears and back, except in the Control group). Mice of the IMQ + BSS group were fed a half-fat, high-sugar diet and stimulated with ice-water swimming every day. Mice of the IMQ + STZ group were fed a half-fat, high-sugar diet and injected with STZ. Mice of the IMQ + STZ + BSS group were subjected to the same treatments as the IMQ + STZ and IMQ + BSS groups. After induction, the mice in each group were observed for vital signs, ear thickness, body weight, and psoriasis area and severity index (PASI) score. The mice were fasted for $12 \mathrm{~h}$ before determination of related laboratory serum indexes. Dorsal skin lesions, aortic arch pathology sections, and signal transducer and activator of transcription 3 (STAT3) were examined by $\mathrm{H} \& \mathrm{E}$ staining and immunohistochemistry. Results. Laboratory indexes in the four model groups were significantly different from those in the Control group $(p<0.01)$; indicators of the IMQ + STZ, IMQ + BSS, and IMQ + STZ + BSS groups showed varying degrees of difference from those of the IMQ group. Conclusions. The established mouse models of psoriasis blood stasis syndrome complicated with glucose and lipid metabolism disorders met the clinical and Chinese Medicine characteristics, and thus they could be used as animal models in future studies of psoriasis complicated with glucose and lipid metabolism disorders.
\end{abstract}

\section{Introduction}

Psoriasis is a clinically common chronic relapsing inflammatory skin disease, and it is called "Baibi" or "Songpixian" in Chinese Medicine (CM). A previous study [1] has shown that patients with psoriasis have obvious local microcirculation disorders and varying degrees of vascular endothelial injury, which cause increased blood viscosity and microvascular disorders [2]. In addition, significantly abnormal lipid metabolism is shown by patients with psoriasis [3]. Elevated cholesterol, triglyceride, and low-density lipoprotein (LDL) levels can accelerate the adhesion and aggregation of platelets as factors affecting psoriasis [4]. Augustin et al. [5] found in a retrospective study that the incidence of metabolic syndrome in patients with psoriasis is much higher than that in healthy people, and metabolic syndrome is a high risk factor for cardiovascular and cerebrovascular diseases. Currently, the available treatment 
methods for psoriasis are mainly based on biological and systematic therapies, supplemented by physical therapy. However, some adverse reactions are associated with the above therapies, such as abnormal glucose and lipid metabolism, which undoubtedly worsen the condition of patients with psoriasis complicated with metabolic disorders $[6,7]$. Therefore, studies on models of psoriasis complicated with metabolic disorders have important significance in the development of drugs for the treatment of psoriasis, which may reduce the incidence of complications and improve the quality of life and survival rate of patients.

In $\mathrm{CM}$, blood is believed to be the disease location of psoriasis, given that the classic CM syndromes of psoriasis include "blood heat," "blood stasis," and "blood deficiency" [8]. Moreover, phlegm, heat, stasis, and asthenia represent the key pathomechanism of metabolic disorders [9]. In the early stage of psoriasis, blood heat is abundant and causes obstruction of the muscle surface. In the later stage, blood heat is concentrated in the blood, and blood circulation is not smooth and turns into static blood. Alternatively, the chronic disease consumes qi and blood, and the deficiency of qi and blood can cause blood stasis, all of which can lead to the occurrence of metabolic syndrome, in which blood stasis is common [10]. Blood stasis syndrome occurs throughout the onset of psoriasis [11]. When patients with psoriasis are in the state of blood stasis syndrome for a long time, static blood is latent in the meridians, viscera, and limbs, forming various symptoms similar to glycolipid metabolism disorders, such as diabetes and metabolic syndrome [12]. At present, there are neither reports nor studies on animal models of psoriasis with blood stasis syndrome complicated with metabolic disorder, thereby hindering the development of new clinical drugs for this disease. Therefore, this study aimed to establish mouse models presenting the characteristics of psoriasis with blood stasis and glycolipid metabolism disorders. IMQ, STZ, and ice-water swimming were used to induce psoriasis with blood stasis syndrome complicated with glycolipid metabolism disorder in mice.

\section{Materials and Methods}

\subsection{Material and Apparatus}

2.1.1. Animals. Forty-five male C57BL/6 mice weighing $25 \pm 3 \mathrm{~g}$ were provided by Shanghai Medical Experimental Animal Center (SCXK Shanghai 2013-0016, Shanghai, China). The animals were maintained under a standard temperature of $23 \pm 2^{\circ} \mathrm{C}$ and 12 -h light-dark cycle. The mice were grouped into three mice per cage with free access to standard diet and water. All procedures were reviewed and approved by the Scientific Research Department of Yueyang Hospital affiliated to Shanghai University of Traditional Chinese Medicine (animal certificate nos. 2015000544758, 2015000546963, and 2015000549944). All procedures were reviewed and approved by the Ethics Committee of Yueyang Hospital affiliated to Shanghai University of Traditional Chinese Medicine (no. 17772).

2.1.2. Groups. All mice were randomly divided into five groups: blank control group (Control group), psoriasis group (IMQ group), psoriasis with metabolic disorders group (IMQ + STZ group), psoriasis with blood stasis syndrome (BSS) group (IMQ + BSS group), and psoriasis with blood stasis syndrome complicated with metabolic disorders group (IMQ + STZ + BSS group), with nine mice in each group.

2.1.3. Experimental Apparatus. Low-density lipoproteincholesterol (LDL-C) and total cholesterol testing kits were purchased from Nanjing Jiancheng Institute of Biological Engineering (Jiangsu, China). Vascular endothelial growth factor (VEGF), endothelin-1 (ET-1), insulin, and mouse C-peptide testing kits were obtained from Shanghai Xinyu Biotechnology Co., Ltd. (Shanghai, China), and the rapid glucose meter was purchased from Bayer (Baiankang 1816, Leverkusen, Germany).

2.1.4. Drugs. IMQ cream was purchased from Sichuan Mingxin Pharmaceutical Co., Ltd. (Sichuan, China; batch number: National Drug Approval Letter H20030128). Norepinephrine (NE) adjusted to $0.1 \mathrm{~g} / 1$ was obtained from Shanghai Wellhope Pharmaceutical Co., Ltd. (Shanghai, China; batch number: National Drug Approval Letter H31021177). STZ powder (batch number: Cas18883-66-4) was purchased from Beijing Boai Port Trade Center (Beijing, China). STZ buffer was configured from liquid A (0.1 M citric acid solution, $1.05 \mathrm{~g}$ constant volume of $50 \mathrm{ml}$ ) and liquid B (0.1 M sodium citrate solution, $1.47 \mathrm{~g}$ constant volume of $50 \mathrm{ml}$ ) at $4^{\circ} \mathrm{C}$, avoiding light preservation, to $10 \mathrm{mg} / \mathrm{ml}$ solution configured to STZ before use. Rabbit antiphosphorylation signal transducers and activators of transcription-3 (STAT3) antibodies (batch number: GR287047-7) were purchased from Abcam Co. (Cambridge, United Kingdom).

2.2. Establishment of Mouse Models. The model establishment methods are shown in Figure 1(a).

2.2.1. IMQ Group. The back hair of the mice was shaved, and IMQ cream was applied to the skin on their ears and back $\left(1 \mathrm{~cm}^{2}\right.$ of skin; $0.01 \mathrm{~g}$ IMQ cream was applied evenly using a rubber hose) once a day for seven days [13]. Photographs were taken daily to record changes in the skin lesion on the back, and the thickness of the ears was measured.

2.2.2. IMQ + STZ Group. Mice were fed a half-fat and highsugar diet $(84.5 \%$ basic feed with $5 \%$ yolk powder, $0.5 \%$ cholesterol, 5\% lard, and 5\% sucrose) for one week. STZ solution was administered via intraperitoneal injection to mice at a dose of $100 \mathrm{mg} / \mathrm{kg}$ once daily for two days, and blood glucose level was measured on the fifth day. An obvious increase in blood glucose level indicated successful model establishment. Otherwise, the dose was administered again on the fifth day until blood glucose level reached $>14.9 \mathrm{mmol} / \mathrm{l}$. The day after blood glucose level reached $>14.9 \mathrm{mmol} / \mathrm{l}$, and IMQ cream was applied to the skin of the ears and back of the mice, with the same method as for the 

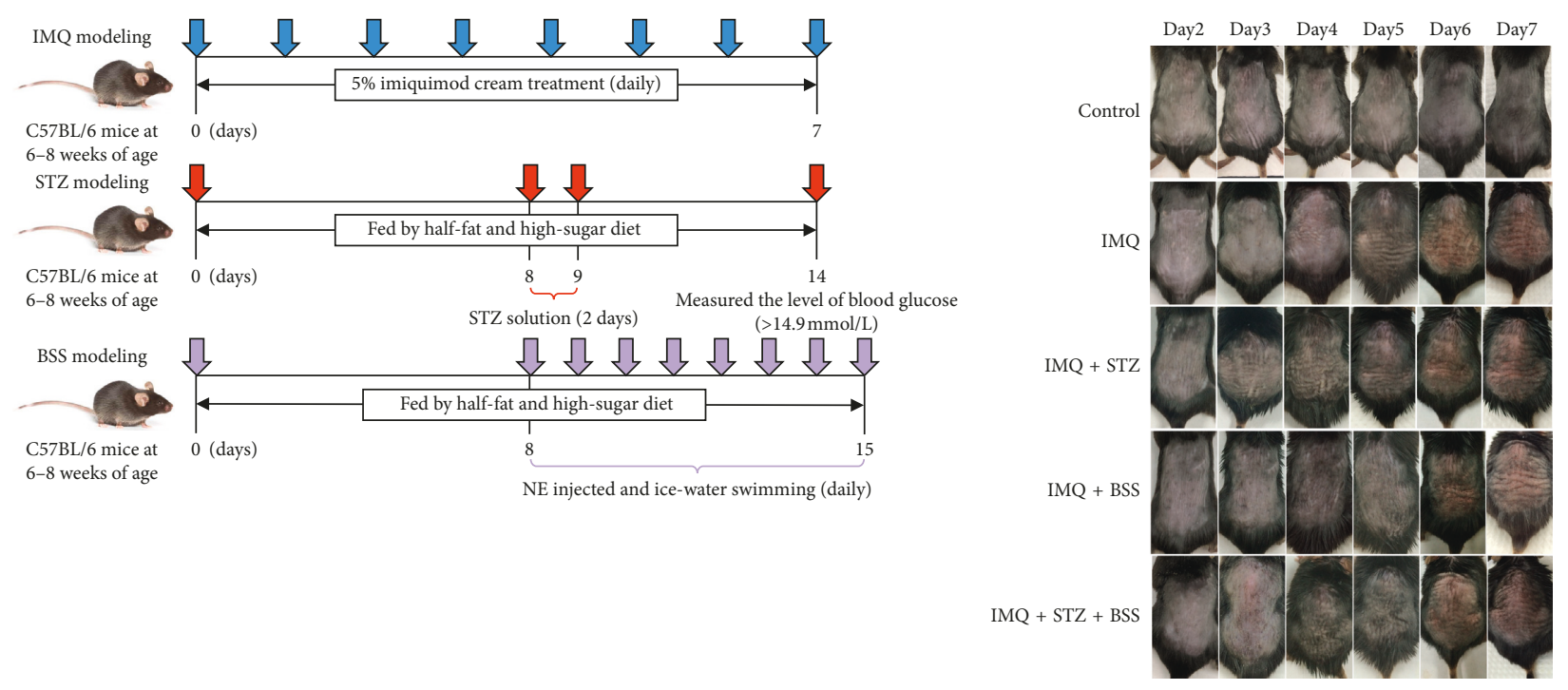

(a)

(b)
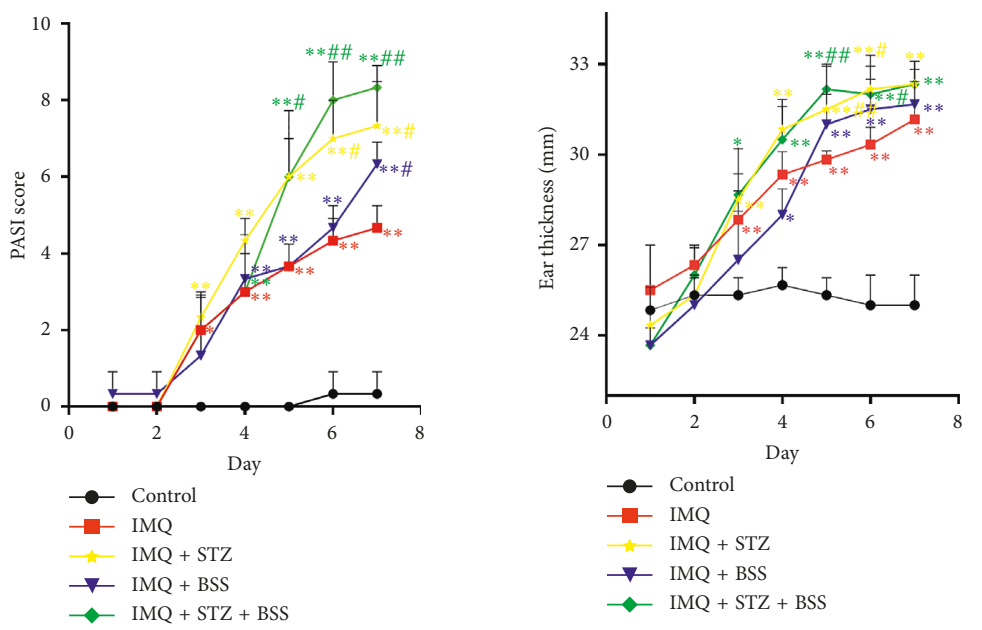

(c)

(d)

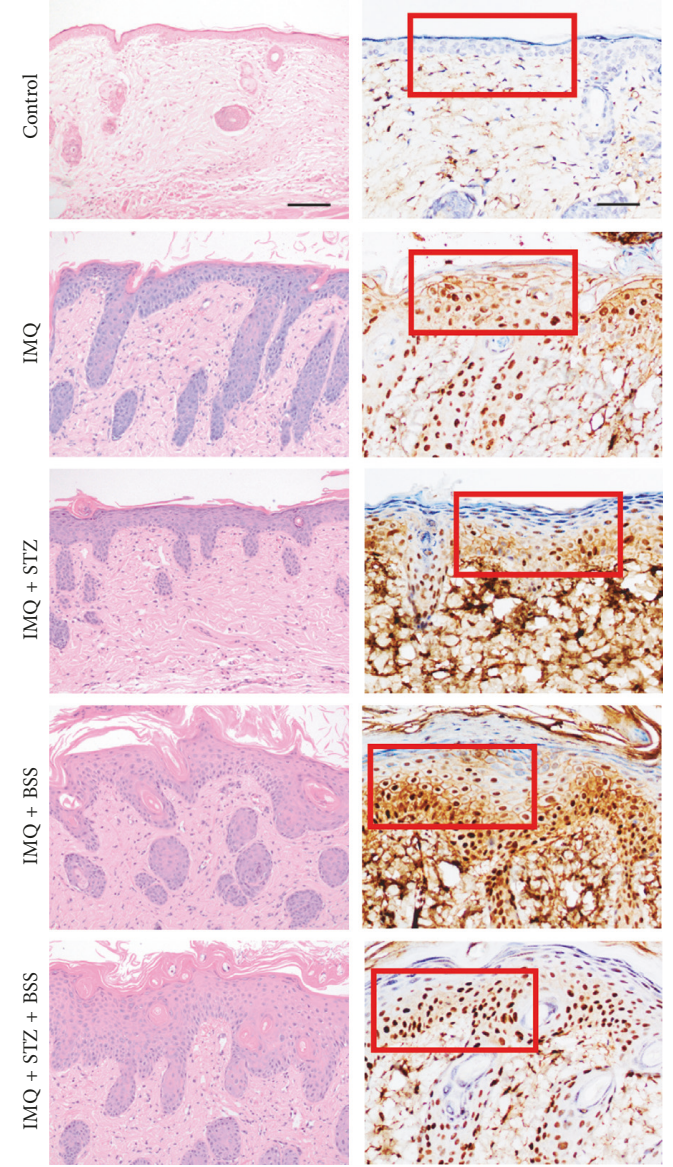

(e)

FIgURE 1: Continued. 


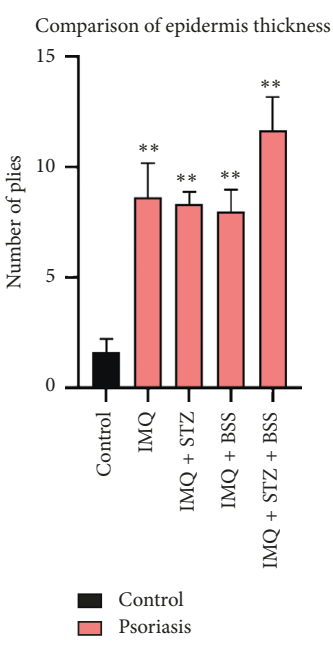

(f)

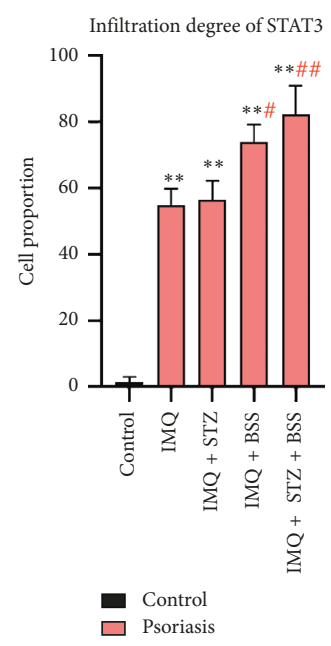

(g)

FIGURE 1: Induction methods and establishment of mouse psoriasis models. (a) Induction methods involving IMQ, STZ, and BSS. (b) After induction, skin lesions on the back of mice in each group were recorded for seven days. (c) After induction, the PASI score of back-skin lesions in each group was determined for seven days. (d) After induction, ear thickness in each group was measured for seven days. (e) Left: pathological sections of mouse back skin (H\&E staining; magnification, $\times 100$; scale bar, $100 \mu \mathrm{M}$ ) showed thickening of the epidermis in each group on the last day of induction. Right: immunohistochemical analysis of mouse back skin (magnification, $\times 200$; scale bar, $50 \mu \mathrm{M}$ ); brown-yellow granule cells indicate STAT3-positive cells. (f) Comparison of epidermis thickness on the last day of induction. (g) Infiltration degree of STAT3 on the last day of induction. Compared with the control group, ${ }^{* *} p<0.01,{ }^{*} p<0.05$; compared with the IMQ group, ${ }^{\# \#} p<0.01,{ }^{\#} p<0.05$.

IMQ group. Starting from the first day of IMQ cream application, the skin lesions were photographed and recorded daily, ear thickness was measured, and body weights were recorded.

2.2.3. IMQ + BSS Group. Mice were fed a half-fat and highsugar diet $(84.5 \%$ basic feed with $5 \%$ yolk powder, $0.5 \%$ cholesterol, 5\% lard, and 5\% sucrose) for one week. NE was administered via intraperitoneal injection in mice at a dose of $0.1 \mathrm{ml} / 100 \mathrm{~g}(0.1 \mathrm{~g} / \mathrm{l} \mathrm{NE}$ was prepared in normal saline). After 15 to $20 \mathrm{~min}$, the mice were induced to swim in ice water at $4^{\circ} \mathrm{C}$ for 5 min once a day for 8 days. On the second day of ice-water swimming, IMQ cream was applied to the skin of the ears and back of the mice, using the same method as for the IMQ group. Starting from the first day of IMQ cream application, skin lesions were photographed and recorded daily, ear thickness was measured, body weights were recorded, and changes in mouse hair color, nails, tongue, and activity were observed every day.

2.2.4. $I M Q+S T Z+B S S$ Group. The psoriasis induction method was the same as for the IMQ group, and the induction methods of blood stasis syndrome and metabolic disorder were the same as for the IMQ + BSS and IMQ + STZ groups, respectively. During the eight consecutive days of model induction, skin lesions were photographed and recorded daily, ear thickness was measured, and body weights were recorded.

2.2.5. Specimen Collection. The experimental cycle was approximately 15 days. The mice were fasted but allowed water for $12 \mathrm{~h}$ before specimen collection. At $8 \mathrm{a} . \mathrm{m}$. on the next day, $2 \mathrm{ml}$ blood was extracted via the eyeball: $1 \mathrm{ml}$ was injected into an anticoagulant test tube, and the other $1 \mathrm{ml}$ was injected into a coagulant test tube. Blood samples were allowed to stand for $4^{\circ} \mathrm{C}$ for $1 \mathrm{~h}$ and then centrifuged in a refrigerated centrifuge at $4^{\circ} \mathrm{C}$ and $1800 \mathrm{rpm} / \mathrm{min}$ for $5 \mathrm{~min}$. The supernatant was then collected and stored in a refrigerator at $-80^{\circ} \mathrm{C}$ until analysis. The mice were sacrificed in a euthanasia box with the flow rate of $\mathrm{CO}_{2}$ into the box maintained at $20 \%$ volume of the box per minute. Specimens of the bilateral ears, back skin, and aortic arch were collected using surgical scissors and fixed in $100 \mathrm{ml} / \mathrm{l}$ paraformaldehyde solution. Paraffin blocks of specimens were then prepared routinely for analysis.

2.2.6. Statistical Method. The data were analyzed by using the SPSS.21.0 statistical software (International Business Machines Corporation, Armonk, NY, USA). The data were expressed as $(\bar{x} \pm s)$. Values of $p<0.05$ indicated a statistical difference, and $p<0.01$ indicated a significant difference. A group sample $t$-test was used for comparison between groups.

\section{Results}

3.1. Characteristics of Back-Skin Lesions and Ear Thickness in the Animal Models. The pathological features of the back and changes in ear thickness at days 2 to 7 were observed in the mice. The IMQ group showed significant thickening of the back skin on the fourth day of induction; on the seventh day of induction, there were obvious scales and erythema on the back. In the IMQ + STZ group, the skin thickened on the second day of induction, and the back skin became hard after 
6 and 7 days of induction, accompanied by patches, peeling, and descaling. This suggested that glucose and lipid metabolism imbalance may accelerate the development of psoriatic lesions. The IMQ + BSS group showed significant psoriatic skin lesions on the fourth day of induction. On the seventh day of induction, the mice showed clinical manifestations of blood stasis: pale and cold back skin, bluish purple tongue, dim eyeball color, petechiae in the tail, and a significant scaly and erythema lesion area on the back skin. The IMQ + STZ + BSS group showed thickening of the back skin on the third day of induction; at the end of induction, the skin lesions on the back were pale, peeling, and desquamated. The mice also showed blood stasis syndrome, which was slightly less severe than that in the IMQ + BSS group. Owing to the slow induction process of blood stasis syndrome, skin lesions in the early stage were not significant. The fastest psoriasis induction process was observed in the $\mathrm{IMQ}+\mathrm{STZ}$ group, and the most prominent psoriatic skin lesions were observed in the IMQ + STZ + BSS group (Figure 1(b)).

The PASI score showed similar results (Figure 1(c)). The PASI score of the back-skin lesions in each group showed varying degrees of changes on the third day of induction. The PASI score of the IMQ + STZ group showed the fastest increase, whereas that of the IMQ + STZ + BSS group was the highest on the seventh day of induction, suggesting that psoriasis complicated with glucose and lipid metabolism disorders may aggravate the primary skin lesions of psoriasis. In addition, the PASI score of the $\mathrm{IMQ}+\mathrm{BSS}$ group increased slightly faster than that of the IMQ group, suggesting that blood stasis syndrome may accelerate the manifestation of primary skin lesions in psoriasis $(p<0.01)$.

IMQ cream was applied to the mouse ears, and mouse ear thickness was measured on the first to seventh day (Figure $1(\mathrm{~d})$ ). We found that mouse ears in the IMQ, $\mathrm{IMQ}+\mathrm{STZ}, \mathrm{IMQ}+\mathrm{BSS}$, and IMQ + STZ + BSS groups were thicker than those in the Control group $(p<0.01)$. Thickening of the mouse ears was the fastest in the $\mathrm{IMQ}+\mathrm{STZ}+\mathrm{BSS}$ group, followed by the IMQ + STZ group. The ears of the IMQ + BSS group were slightly thicker than those of the IMQ group, suggesting the same conclusion as that suggested by the PASI score.

3.2. Histopathological Features of Back-Skin Lesions. Mouse back skin collected on day 7 was stained with $\mathrm{H} \& \mathrm{E}$ and observed at 100× magnification (Figure 1(e), left). The IMQ, IMQ + STZ, IMQ + BSS, and IMQ + STZ + BSS groups showed psoriatic pathological changes compared with the Control group ( $p<0.01$; Figure $1(\mathrm{f}))$, such as epidermal hyperkeratosis or hyperkeratosis, epidermal cluster-like hyperplasia, dermal papillary extension, dermal papillary capillary dilation, congestion, and inflammatory cell infiltration around the upper dermal vessels. The back-skin lesions of the Control group showed epidermal and spinous layers arranged in a regular order, and the spinous layer was composed of 3-5 layers of polygonal cells, with clear boundaries of the true epidermis, no inflammatory infiltration of lymphocytes, and no telangiectasias or congestion.

Using immunohistochemical staining and microscopy at 200x magnification (Figure 1(e), right), we observed cells with brown-yellow granules in the cytoplasm and nucleus, which were STAT3-positive cells. There were almost no STAT3-positive cells in the dermis of the Control group. However, compared with the Control group, the IMQ, $\mathrm{IMQ}+\mathrm{STZ}, \mathrm{IMQ}+\mathrm{BSS}$, and IMQ + STZ + BSS groups showed significantly increased STAT3 expression $(p<0.01$; Figure $1(\mathrm{~g}))$. We examined the histogram at $\times 200$ magnification and calculated the score of STAT3-positive cells in each group: light yellow, 1 point; yellow and dark yellow, 2 points; and brown-yellow, 3 points. The score of STAT3positive cells was the highest in the IMQ $+\mathrm{STZ}+\mathrm{BSS}$ group; STAT3-positive cells in the IMQ + STZ + BSS and IMQ + BSS groups were darker than those in the IMQ group $(p<0.05)$, suggesting that glucose and lipid metabolism disorders and blood stasis syndrome promoted the proliferation and differentiation of keratinocytes (KCs), and that the expression of STAT3 was positively correlated with PASI score.

\subsection{Indexes of Glycolipid Metabolism in Animal Models}

3.3.1. Changes in Serum Insulin, C-Peptide, and Blood Glucose Levels. Compared with the Control group at the end of induction, serum insulin levels significantly increased in the IMQ, IMQ + STZ, and IMQ + STZ + BSS groups $(p<0.01)$, but significantly decreased in the IMQ + BSS group $(p<0.01)$. Compared with those in the Control group, serum C-peptide levels significantly decreased in the $\mathrm{IMQ}+\mathrm{STZ}$, IMQ + BSS, and IMQ + STZ + BSS groups $(p<0.01)$, suggesting that islet dysfunction occurred in varying degrees in each model group after induction. Compared with those of the IMQ group, blood glucose levels significantly increased in the IMQ + STZ and $\mathrm{IMQ}+\mathrm{STZ}+\mathrm{BSS}$ groups $(p<0.01)$, but significantly decreased in the IMQ + BSS group $(p<0.01)$, suggesting that STZ induction increased blood glucose level. Moreover, blood glucose level in the IMQ + BSS group might have been too low owing to insufficient insulin secretion (Figure 2(a)).

3.3.2. Changes in Weight, Total Cholesterol, Triglyceride, $H D L$, and LDL Levels. On the seventh day of induction, body weights of animals significantly decreased in the IMQ $(p<0.05), \quad \mathrm{IMQ}+\mathrm{BSS} \quad(p<0.05)$, and IMQ + STZ + BSS $(p<0.01)$ groups, and insignificantly decreased in the $\mathrm{IMQ}+\mathrm{STZ}$ group. Mouse body weights in the IMQ, $I M Q+S T Z, \quad I M Q+B S S$, and IMQ + STZ + BSS groups slightly increased at the fifth and sixth days of induction, which may be related to the induction treatments. At the end of the induction period, weight loss was the most obvious in the IMQ + BSS group, followed by the IMQ + STZ + BSS and $\mathrm{IMQ}+\mathrm{STZ}$ groups, and the least obvious in the IMQ group (Figure 2(b)).

Compared with those in the Control group at the end of induction, the levels of total cholesterol, triglycerides, and 

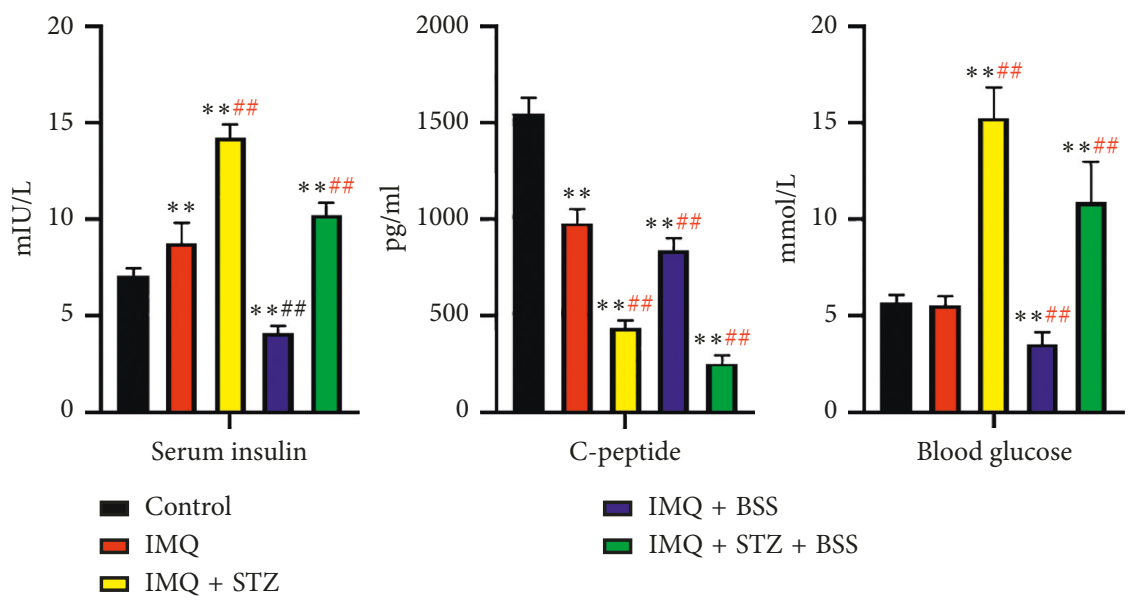

(a)

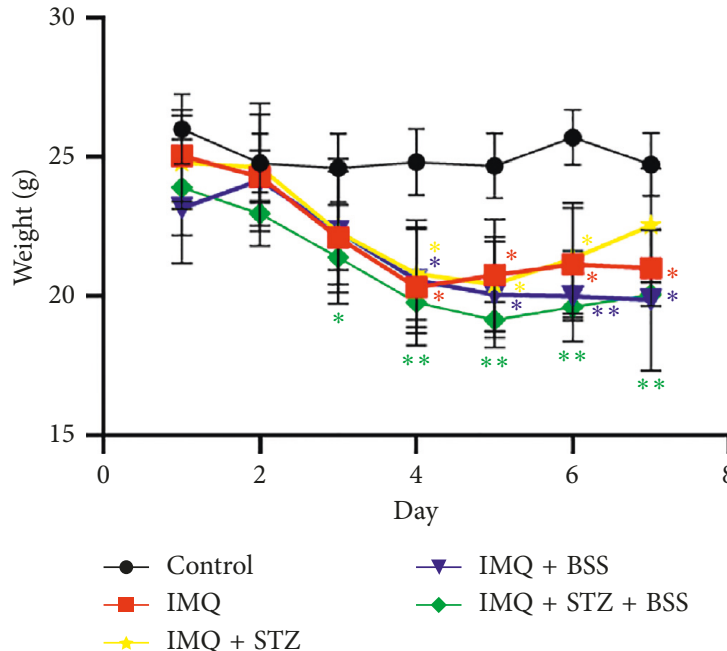

(b)
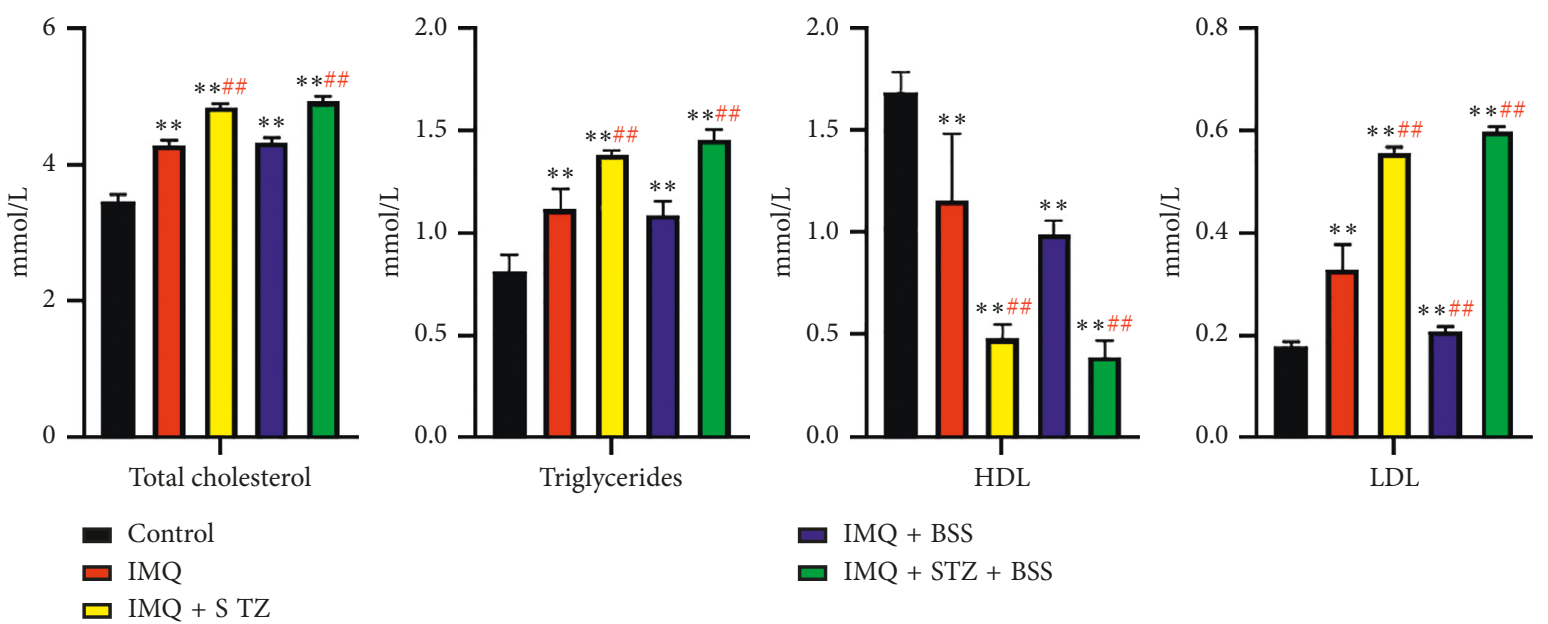

(c)

Figure 2: Levels of indicators of glucose and lipid metabolism. (a) Serum insulin, C-peptide, and blood glucose levels of each group on the last day of induction. (b) Body weights of each group measured for 7 days of IMQ induction. (c) Total cholesterol, triglyceride, HDL, and LDL levels of each group on the last day of induction. Compared with the control group, ${ }^{* *} p<0.01,{ }^{*} p<0.05$; compared with the IMQ group, ${ }^{\# \#} p<0.01,{ }^{\#} p<0.05$. 
LDL significantly increased in each model group $(p<0.01)$, whereas the levels of HDL were significantly decreased in each model group $(p<0.01)$, indicating that lipid metabolism disorders were prevalent in each model group (Figure 2(c)). However, the levels of total cholesterol, triglycerides, and LDL in the IMQ + STZ and IMQ + STZ + BSS groups were significantly higher than those in the IMQ group $(p<0.01)$, accompanied by decreased HDL levels $(p<0.01)$. This result suggested that compared with the Control group, the IMQ group had a certain degree of lipid metabolism disorder, and the model groups induced with STZ showed more severe lipid metabolism disorder than the IMQ group.

3.3.3. Changes in Whole Blood Viscosity. Compared with that in the Control group, the whole blood viscosity index of each model group was significantly increased, suggesting that psoriasis induction can lead to an increase in whole blood viscosity. Compared with that in the IMQ group, the whole blood viscosity of the IMQ + STZ + BSS group significantly decreased in both medium and high shear $(p<0.01)$. The high-shear whole blood viscosity level significantly increased in the IMQ + STZ and IMQ + BSS groups $(p<0.05)$, indicating that blood stasis syndrome as well as glucose and lipid metabolism disorders helped increase blood viscosity index (Figure 3(a)).

3.3.4. Changes in VEGF and ET-1 Levels. Compared with those in the control group, VEGF and ET-1 levels were significantly increased in each model group at the end of induction $(p<0.01)$, especially in the IMQ + STZ and $\mathrm{IMQ}+\mathrm{STZ}+\mathrm{BSS}$ groups, suggesting that the three interventions (psoriasis, blood stasis syndrome, and glucose and lipid metabolism disorders) caused different degrees of microcirculation disorders. In addition, compared with that in the IMQ group, VEGF level in the IMQ+STZ, $\mathrm{IMQ}+\mathrm{BSS}$, and IMQ + STZ + BSS groups increased significantly $(p<0.01)$. The levels of ET- 1 in the IMQ + STZ and IMQ + STZ + BSS groups significantly increased, further suggesting that glucose and lipid metabolism disorders and blood stasis syndrome aggravated psoriasis (Figure 3(b)).

3.3.5. Changes in Dissection of Aortic Arch. On the seventh day of induction, the aortic arch was dissected from the mice and stained with H\&E (Figure 3(c)). Microcopy analysis at $\times 100$ magnification showed that the aortic arch wall markedly thickened in the IMQ + STZ, IMQ + BSS, and $\mathrm{IMQ}+\mathrm{STZ}+\mathrm{BSS}$ groups, and the intima in the IMQ + BSS group had a significantly granular bright red lining of visible lipid deposition and scattered calcification points. The aortic arch intima of the IMQ + STZ and IMQ + STZ + BSS groups showed accumulated lipid droplets, whereas that of the IMQ group showed neither lipid deposition nor lipid droplet accumulation. In addition, the aortic vessel lumen in the IMQ + STZ, IMQ + BSS, and IMQ + STZ + BSS groups was narrower and smaller than that in the Control group, with connective tissue hyperplasia on the outside of the wall.

\section{Discussion}

The idea of combining diseases and syndromes of CM has been used to study psoriasis animal model induction. At present, there are two published Chinese studies on the induction of blood stasis in psoriasis $[14,15]$, both of which used the ice-water swimming method to simulate blood stasis syndrome in combination with the characteristics of psoriasis skin lesions. The induction method is relatively mature, which induced the psoriasis-like lesions, and the blood stasis syndrome score was significantly higher than control. However, induction methods of specific syndromes of psoriasis complicated with other diseases (disorder of glucose and lipid metabolism) have not been reported.

Modern clinical research has demonstrated the existence of glucose and lipid metabolism disorders in patients with psoriasis, which is more likely to be complicated with fatty liver and diabetes $[16,17]$. In psoriatic patients, lipid metabolism disorder is prone to cause metabolic syndrome [18], which is an aggregation of multiple clinical chronic disease risk factors, and its main clinical outcomes are cardiovascular and cerebrovascular diseases and diabetes. A prospective cohort study [19] showed that metabolic syndrome has a direct impact on the total mortality of the population and mortality due to cardiovascular disease, which can be used as an indicator to predict the total mortality of the population. Therefore, the significance of studying psoriasis with metabolic disorders cannot be ignored, and animal experiments are indispensable. A previous study [20] confirmed the existence of metabolic disorders in IMQ-induced psoriasis animal models; hyperglycemia has been shown to be closely associated with psoriasis, mainly through the expression of IL-17. In this study, ice-water swimming was used to simulate blood stasis syndrome, and STZ was used to induce type II diabetes; this was the first attempt to explore psoriasis with blood stasis syndrome complicated with glucose and lipid metabolism disorder.

Our results showed that, compared with the Control group, thickening of mouse dorsal skin, increased erythema and scaling, and increased PASI score were observed in the groups treated with IMQ cream, indicating successful establishment of psoriasis models. Half-fat diet was used to induce obesity and insulin resistance in mice [21], and intraperitoneal injection of a chain urea toxin (STZ) was applied to destruct pancreatic islet $\beta$ cells in the mice [22], thus generating a condition similar to human type II diabetes. In the established mouse models, laboratory indicators, including C-peptide, glucose, total cholesterol, triglyceride, and LDL levels, increased, whereas HDL levels decreased, indicating the successful establishment of mouse models of psoriasis with glucolipid metabolic disorder. Notably, serum insulin levels were high in groups treated with STZ (Figure 2(a)). STZ-induced diabetic modeling is an accepted method in which pancreatic islet $\beta$ cells are destroyed, leading to islet dysfunction and insufficient insulin secretion. In our study, insulin levels in the non-STZ groups were slightly lower than those in the STZ groups, and blood glucose levels of STZ mice were elevated significantly, 

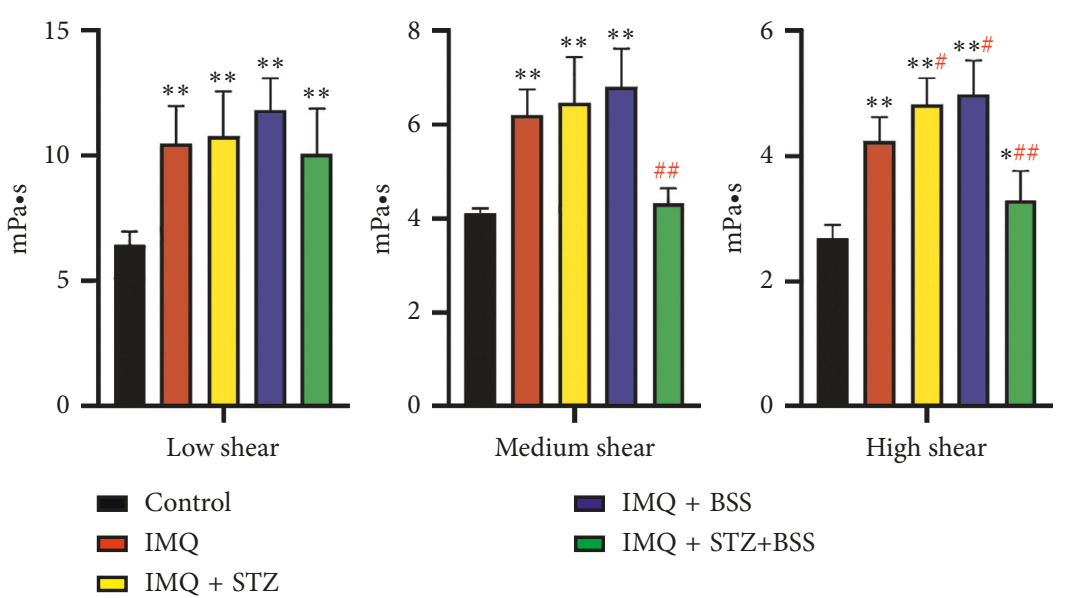

(a)

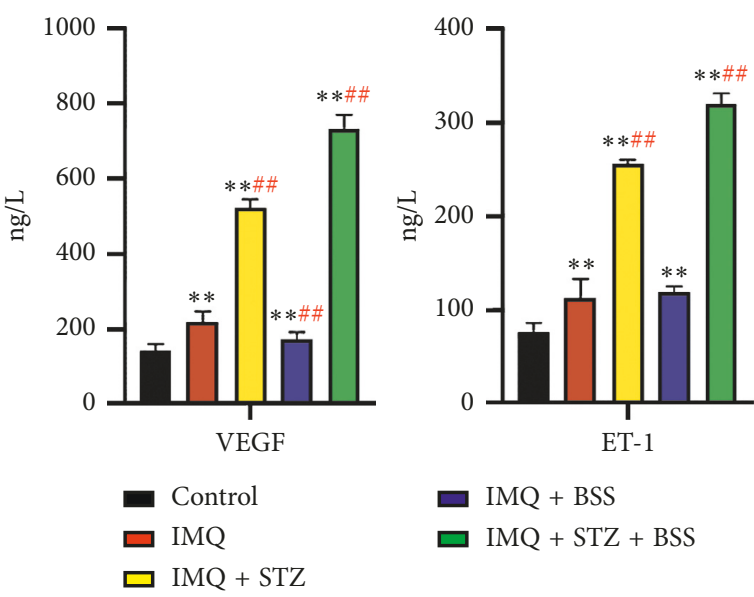

(b)

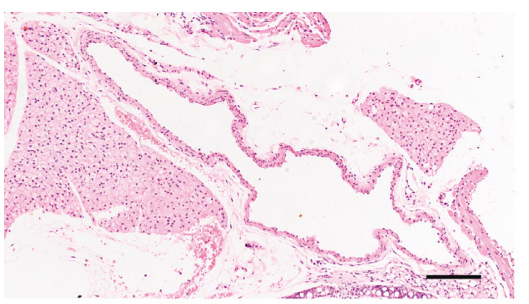

Control

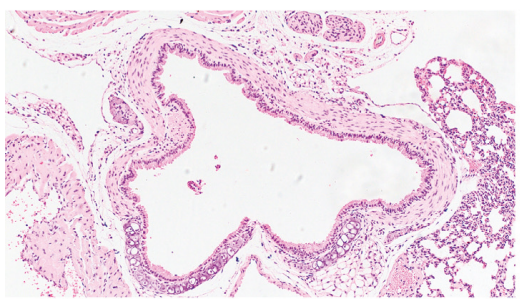

$\mathrm{IMQ}+\mathrm{BSS}$

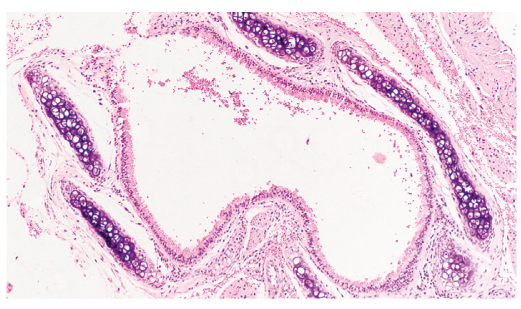

IMQ

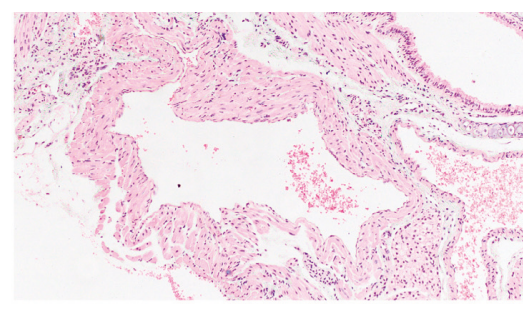

IMQ + STZ

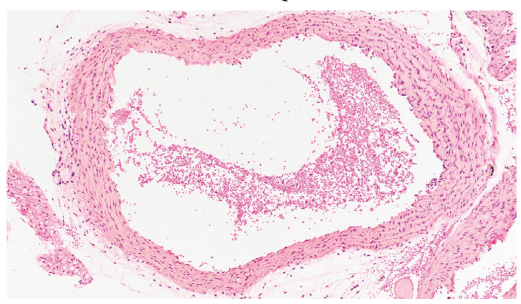

$I M Q+S T Z+B S S$

(c)

FIGURE 3: Levels of cardiovascular indicators. (a) Whole blood viscosity levels of each group on the last day of induction. (b) VEGF and ET-1 levels of each group on the last day of induction. (c) Pathological sections of mouse aortic arch from each group (H\&E staining; magnification, $\times 100$; scale bar, $100 \mu \mathrm{M})$. Compared with the control group, ${ }^{* *} p<0.01,{ }^{*} p<0.05$; compared with the IMQ group, ${ }^{\# \#} p<0.01,{ }^{\#} p<0.05$.

which led to insulin resistance and induce type II diabetes. These results may indicate that our IMQ-STZ models were undergoing a transition from an insulin-resistant state to induce type II diabetes [23]. The blood stasis group received daily injection of NE to induce chronic endothelial cell injury and establish chronic blood stasis models; moreover, 
ice-water swimming resulted in Yang deficiency and warm blood, which led to blood stasis. The laboratory indexes after induction showed that whole blood viscosity increased, suggesting the successful establishment of blood stasis models [24].

From the microscopic perspective, increased VEGF and ET-1 indexes verified that the internal environment of the IMQ, IMQ + STZ, IMQ + BSS, and IMQ + STZ + BSS groups all presented different degrees of microcirculation disorders. The pathological sections of the dorsal skin showed thickening of the spinous layer, and inflammatory infiltration was most significant in the IMQ + STZ + BSS group, whereas the IMQ + STZ and IMQ + BSS groups showed the same trend of thickening and infiltration, suggesting that both blood stasis syndrome and glucose and lipid metabolism disorders aggravated the skin lesions of psoriasis. Pathological sections showed that the intima of the aortic arch in the IMQ + STZ and IMQ + STZ + BSS groups presented lipid droplet aggregation, accompanied by narrowing of the vascular lumen and hyperplasia of connective tissue on the outside of the arch wall, whereas the intima of the aortic arch in the IMQ + BSS group showed lipid deposition and scattered calcification points, suggesting that both blood stasis syndrome and glucose and lipid metabolism disorders induced aortic blood flow obstruction.

STAT3, a specific index of psoriatic KC proliferation [25], is mainly expressed in epidermis layers (the base layer, stratum spinosum, granular layer, and transparent layer) other than in the corneous layer. It has been shown that the mRNA expression of STAT3 in psoriasis patients with BSS was significantly higher than that in healthy people [26]. Immunohistochemical analysis results in the IMQ + STZ + BSS group showed a significantly increased number of STAT3positive cells; the IMQ, IMQ + STZ, and IMQ + BSS groups also showed an increased number of STAT3-positive cells, to different extents. Therefore, it was suggested that IMQ cream treatment led to abnormal proliferation of KCs, and that glucose and lipid metabolism disorder and blood stasis syndrome aggravated psoriasis symptoms.

From the perspective of CM, blood stasis is a key to the pathogenesis of psoriasis $[10,27]$. Blood stasis syndrome is also a common syndrome of heart disease, diabetes, vertigo, and other diseases in CM; therefore, studying blood stasis syndrome complicated with glucose and lipid metabolism disorders is of great significance. Previous studies by our research group have found that psoriasis is related to the occurrence of hyperuricemia and chronic obstructive pulmonary disease $[28,29]$, and high-intensity exercise reduces the prevalence of psoriasis [30]. In this study, we compared models of psoriasis with blood stasis syndrome, psoriasis complicated with glucose and lipid metabolism disorders, and psoriasis complicated with both blood stasis syndrome and glucolipid metabolic disorder in terms of aortic tissue pathology, hemorheology, VEGF and ET-1 levels, and other indexes. Our results were close to those observed in clinical practice, as expected, and close to those in human diseases. Thus, our models could be used to explore the pathogenesis of psoriasis and serve as an experimental basis of pharmacodynamics research.

\section{Conclusions}

We established mouse models of psoriasis with blood stasis syndrome complicated with glucose and lipid metabolism disorders, which presented the clinical characteristics specified in CM. Thus, our models could be used in studies investigating psoriasis complicated with glucose and lipid metabolism disorders.

\section{Data Availability}

All data generated during this study are included in this published article and its supplementary information files.

\section{Conflicts of Interest}

The authors declare that there are no conflicts of interest regarding the publication of this article.

\section{Authors' Contributions}

Ying Luo and Yi Ru contributed equally. Ying Luo, XL, and BL conceptualized the study. Ying Luo and YR performed data curation. XL, Ying Luo, and HL carried out experimental treatment. $\mathrm{BL}, \mathrm{LK}$, and $\mathrm{MX}$ were responsible for funding acquisition. YR, MX, and LL investigated the study. Ying Luo, Yue Luo, and SH were responsible for methodology. XL, BL, and XC were involved in project administration. JS, Yue Luo, and XF provided the resources. YR and Ying Luo provided the software. BL and XL supervised the study. HZ, LK, and XS validated the study. RY and Ying Luo prepared the original draft. RY, Ying Luo, and XL reviewed and edited the manuscript. All authors have read and approved the manuscript.

\section{Acknowledgments}

The study was supported by NSFC of China (grant nos. 81874470 and 81973860), National Key Research and Development Program of China (grant no. 2018YFC1705305), Shanghai Development Office of TCM (grant nos. ZY (20182020)-FWTX-1008, ZY (2018-2020)-CCCX-2004-08, and ZY (2018-2020)-FWTX-4010), Development Fund for Shanghai Talents (grant no. 2017047), Young Talent Supporting Program of China Association of Traditional Chinese Medicine (grant no. CACM-2017-QNRC2-(B05)), and Shanghai Natural Science Foundation of China (grant no. 19ZR1458700).

\section{References}

[1] I. Ikonomidis, G. Makavos, E. Papadavid et al., "Similarities in coronary function and myocardial deformation between psoriasis and coronary artery disease: the role of oxidative stress and inflammation," Canadian Journal of Cardiology, vol. 31, no. 3, pp. 287-295, 2015.

[2] P. M. Lowe, M. L. Lee, C. J. Jackson, S. S. To, A. J. Cooper, and L. Schrieber, "The endothelium in psoriasis," British Journal of Dermatology, vol. 132, no. 4, pp. 497-505, 1995. 
[3] A. Pietrzak, A. Michalakstoma, G. Chodorowska et al., "Lipid disturbances in psoriasis: an update," Mediators of Inflammation, vol. 2010, Article ID 535612, 13 pages, 2010.

[4] B. M. Akkara Veetil, E. L. Matteson, H. Maradit-Kremers, M. T. McEvoy, and C. S. Crowson, "Trends in lipid profiles in patients with psoriasis: a population-based analysis," $B M C$ Dermatology, vol. 12, no. 1, p. 20, 2012.

[5] M. Augustin, K. Reich, G. Glaeske, I. Schaefer, and M. Radtke, "Co-morbidity and age-related prevalence of psoriasis: analysis of health insurance data in Germany," Acta Dermato Venereologica, vol. 90, no. 2, pp. 147-151, 2010.

[6] Q. Zheng, W. Jiang, X. Sun et al., "Total glucosides of paeony for the treatment of psoriasis: a systematic review and metaanalysis of randomized controlled trials," Phytomedicine, vol. 62, Article ID 152940, , 2019.

[7] C. Akçali, E. H. Guven, N. Kirtak, H. S. Inaloz, O. Ozgoztasi, and U. Guvenc, "Serum concentrations of interleukin-2 and tumour necrosis factor- $\alpha$ under cyclosporine versus acitretin treatment in plaque-type psoriasis," Journal of International Medical Research, vol. 42, no. 5, pp. 1118-1122, 2014.

[8] Y. Li, X. Zhu, A. Bensussan et al., "Herbal medicine for hot flushes induced by endocrine therapy in women with breast cancer: a systematic review and meta-analysis," EvidenceBased Complementary and Alternative Medicine, vol. 2016, Article ID 1327251, 11 pages, 2016.

[9] M. M. Ko, S. Jang, and J. Jung, "Herbal medicines for metabolic diseases with blood stasis: a protocol for a systematic review and meta-analysis," Medicine, vol. 98, no. 8, Article ID e14543, 2019.

[10] C. J. Lu, J. W. Deng, L. Li et al., "Application of metabolomics on diagnosis and treatment of patients with psoriasis in Traditional Chinese Medicine," Biochimica et Biophysica Acta, vol. 1844, no. 1, pp. 280-288, 2013.

[11] S. Meng, Z. Lin, Y. Wang et al., "Psoriasis therapy by Chinese medicine and modern agents," Chinese Medicine, vol. 13, no. 1, p. 16, 2018.

[12] S. Y. Lin, S. M. Li, and J. F. Fang, "Discussion on etiology and pathogenesis of metabolic syndrome," New Chinese Medical Journal, vol. 42, pp. 1-2, 2010.

[13] D. F. L. Van, S. Mourits, J. S. Voerman et al., "Imiquimodinduced psoriasis-like skin inflammation in mice is mediated via the IL-23/IL-17 axis," Journal of Immunology, vol. 182, no. 9, pp. 5836-5845, 2009.

[14] M. Zhou, Q. Y. Wu, and H. R. Chen, "Discussion on the establishment of animal model of psoriasis with blood stasis syndrome," Liaoning Journal of Traditional Chinese Medicine, vol. 37, pp. 1161-1163, 2010.

[15] D. C. Fan, S. G. Deng, C. J. Lu et al., "Establishment and evaluation of animal models of psoriasis caused by blood stasis," Lishizhen Medicine and Materia Medica Research, vol. 26, pp. 2812-2814, 2015.

[16] A. Olveira, P. Herranz, and M. L. Montes, "Psoriasis and fatty liver: a harmful synergy," Revista Española de Enfermedades Digestivas, vol. 111, pp. 314-319, 2019.

[17] L. Jacob and K. Kostev, "Psoriasis risk in patients with type 2 diabetes in German primary care practices," Primary Care Diabetes, vol. 11, no. 1, pp. 52-56, 2017.

[18] T. J. Love, A. A. Qureshi, E. W. Karlson, J. M. Gelfand, and H. K. Choi, "Prevalence of the metabolic syndrome in psoriasis," Archives of Dermatology, vol. 147, no. 4, pp. 419-424, 2011.

[19] J. Sundström, U. Risérus, L. Byberg, B. Zethelius, H. Lithell, and L. Lind, "Clinical value of the metabolic syndrome for long term prediction of total and cardiovascular mortality: prospective, population based cohort study," BMJ, vol. 332, no. 7546, pp. 878-882, 2006.

[20] K. Ikumi, M. Odanaka, H. Shime et al., "Hyperglycemia is associated with psoriatic inflammation in both humans and mice," Journal of Investigative Dermatology, vol. 139, no. 6, pp. 1329-1338.e7, 2019.

[21] K. Srinivasan, P. S. Patole, C. L. Kaul, and P. Ramarao, "Reversal of glucose intolerance by by pioglitazone in high fat diet-fed rats," Methods and Findings in Experimental and Clinical Pharmacology, vol. 26, no. 5, pp. 327-333, 2004.

[22] Y. Li, T. Hansotia, B. Yusta, F. Ris, P. A. Halban, and D. J. Drucker, "Glucagon-like peptide-1 receptor signaling modulates $\beta$ cell apoptosis," Journal of Biological Chemistry, vol. 278, no. 1, pp. 471-478, 2003.

[23] D. K. Arulmozhi, R. Kurian, S. L. Bodhankar, and A. Veeranjaneyulu, "Metabolic effects of various antidiabetic and hypolipidaemic agents on a high-fat diet and multiple low-dose streptozocin (MLDS) mouse model of diabetes," Journal of Pharmacy and Pharmacology, vol. 60, no. 9, pp. 1167-1173, 2010.

[24] J.-X. Zhang, Y. Feng, Y. Zhang, Y. Liu, S.-D. Li, and M.-H. Yang, "Hemorheology index changes in a rat acute blood stasis model: a systematic review and meta-analysis," African Journal of Traditional, Complementary and Alternative Medicines, vol. 14, no. 4, pp. 96-107, 2017.

[25] S. Sano, K. S. Chan, and J. DiGiovanni, "Impact of Stat3 activation upon skin biology: a dichotomy of its role between homeostasis and diseases," Journal of Dermatological Science, vol. 50, no. 1, pp. 1-14, 2008.

[26] B. Fan, X. Li, K. Ze et al., "Expression of T-helper 17 cells and signal transducers in patients with psoriasis vulgaris of bloodheat syndrome and blood-stasis syndrome," Chinese Journal of Integrative Medicine, vol. 21, no. 1, pp. 10-16, 2015.

[27] Y. Ru, X. N. Yan, S. Q. Yang et al., "Oral taodan granules for mild-to-moderate psoriasis vulgaris: protocol for a randomized, double-blind, multicenter clinical trial," Annals of Translational Medicine, vol. 7, no. 18, p. 488, 2019.

[28] X. Li, X. Miao, H. Wang et al., "Association of serum uric acid levels in psoriasis: a systematic review and meta-analysis," Medicine, vol. 95, no. 19, Article ID e3676, 2016.

[29] X. Li, L. J. Kong, F. L. Li et al., "Association between psoriasis and chronic obstructive pulmonary disease: a systematic review and meta-analysis," PLoS One, vol. 10, Article ID e0145221, 2015.

[30] Q. Zheng, X. Y. Sun, X. Miao et al., “Association between physical activity and risk of prevalent psoriasis: a MOOSEcompliant meta-analysis," Medicine, vol. 97, no. 27, p. e11394, 2018. 


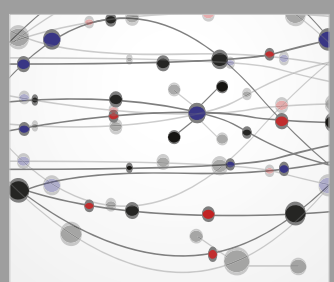

The Scientific World Journal
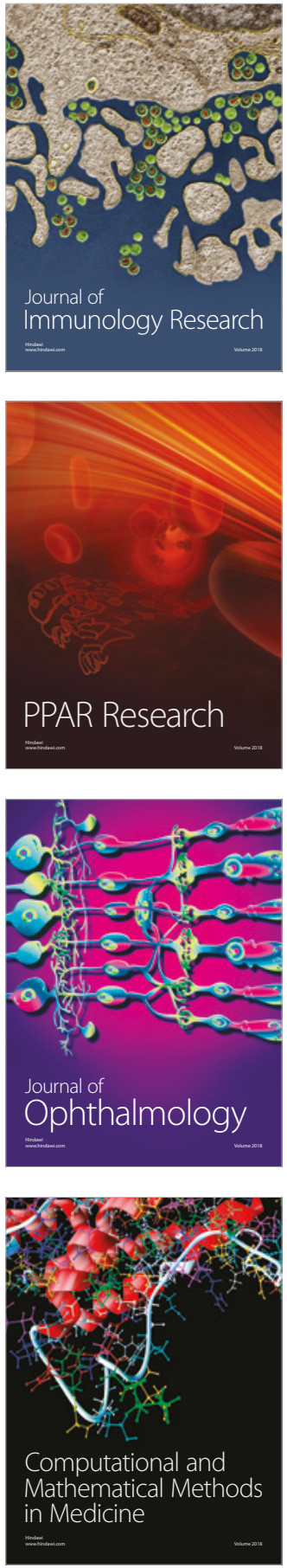

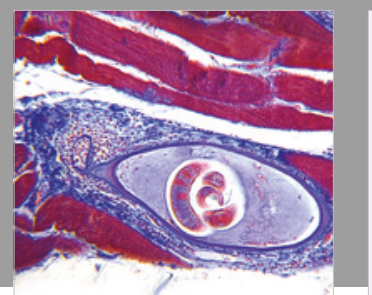

Gastroenterology Research and Practice

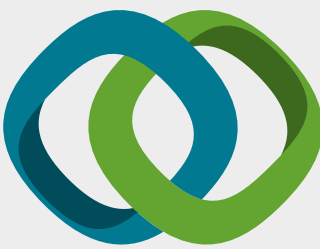

\section{Hindawi}

Submit your manuscripts at

www.hindawi.com
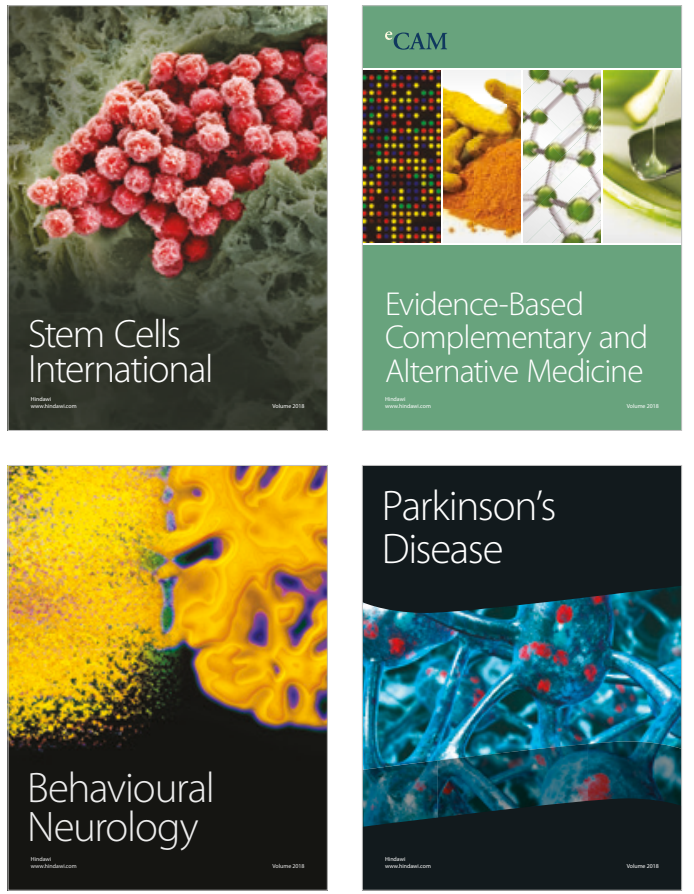

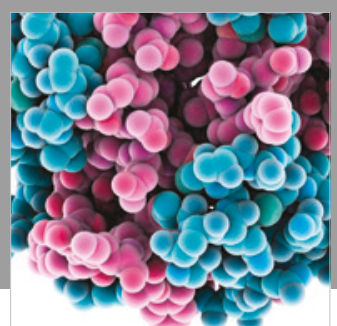

ournal of

Diabetes Research

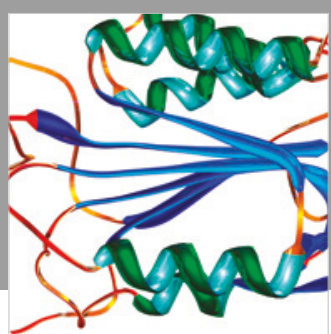

Disease Markers
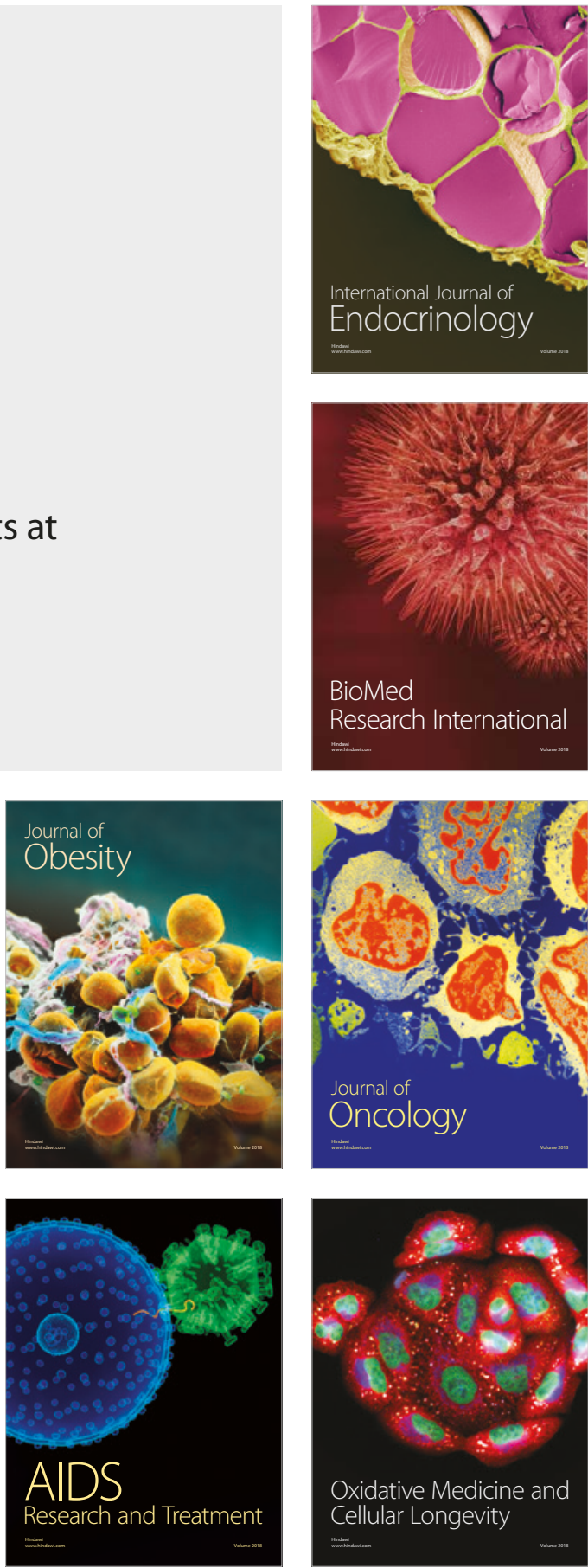